\title{
THE REVERSED POLARITY STRUCTURES OF CHROMOSPHERIC MAGNETIC FIELDS IN ACTIVE REGIONS
}

\author{
WEI LI, GUOXIANG AI, HONGQI ZHANG AND JIMIN CHEN \\ Beijing Astronomical Observatory, Chinese Academy of Sciences, Beijing \\ 100080, China
}

\begin{abstract}
The reversed polarity structures of chromospheric magnetic fields are magnetic gulfs and islands of opposite polarity relative to the underlying photospheric fields. In this paper data were analyzed from the Solar Magnetic Field Telescope of the Huairou Solar Observing Station (HSOS) in Beijing. From more than 300 pairs of photospheric magnetograms (in FeI $\lambda 5324.19 \AA$ ) and relevant chromospheric magnetograms (in $\mathrm{H} \beta \lambda 4861.34 \AA$ ), the reality of the reversed polarity structures is demonstrated. According to an analysis of the fine structures of the magnetic fields in the two layers of active regions, we found that there are probably four different types.
\end{abstract}

\section{LOCALIZED CHROMOSPHERIC POLARITY REVERSALS}

It is possible to make magnetograms with both the $\mathrm{H} \beta \lambda 4861.34 \AA$ chromospheric and FeI $\lambda 5324.19 \AA$ photospheric lines, using the Solar Magnetic Field Telescope at HSOS of Beijing. We analyzed 55 active regions with more than 300 pairs of photospheric and chromospheric magnetograms. We found that the magnetic field peak locations are not entirely coincident between the photospheric and chromospheric observations. Sometimes chromospheric and photospheric magnetograms at the same location of the solar surface showed opposite polarities. Such localized chromospheric polarity reversals were confirmed in 37 active regions. The pattern of the reversed polarity structures of chromospheric magnetic fields usually looks like a gulf or island on contour maps. The reversed structures of chromospheric magnetic fields may be classified in four types (A, $\mathrm{B}, \mathrm{C}$ and $\mathrm{D}$ ), according to the pattern of the structures in the magnetogram. These types are specifically described as follows:

Type A: Chromospheric magnetic islands of opposite polarity corresponding to strong photospheric fields located either near the main $B_{\|}=0$ line or near peaks of the photospheric magnetic field (generally where there is a sunspot umbra or the edge of an umbra). For example, in Figure 1, the marks $A_{1}, A_{2}, A_{3}$ and $A_{4}$ show the locations for type A reversed structures of chromospheric magnetic fields relative to the photospheric fields. 


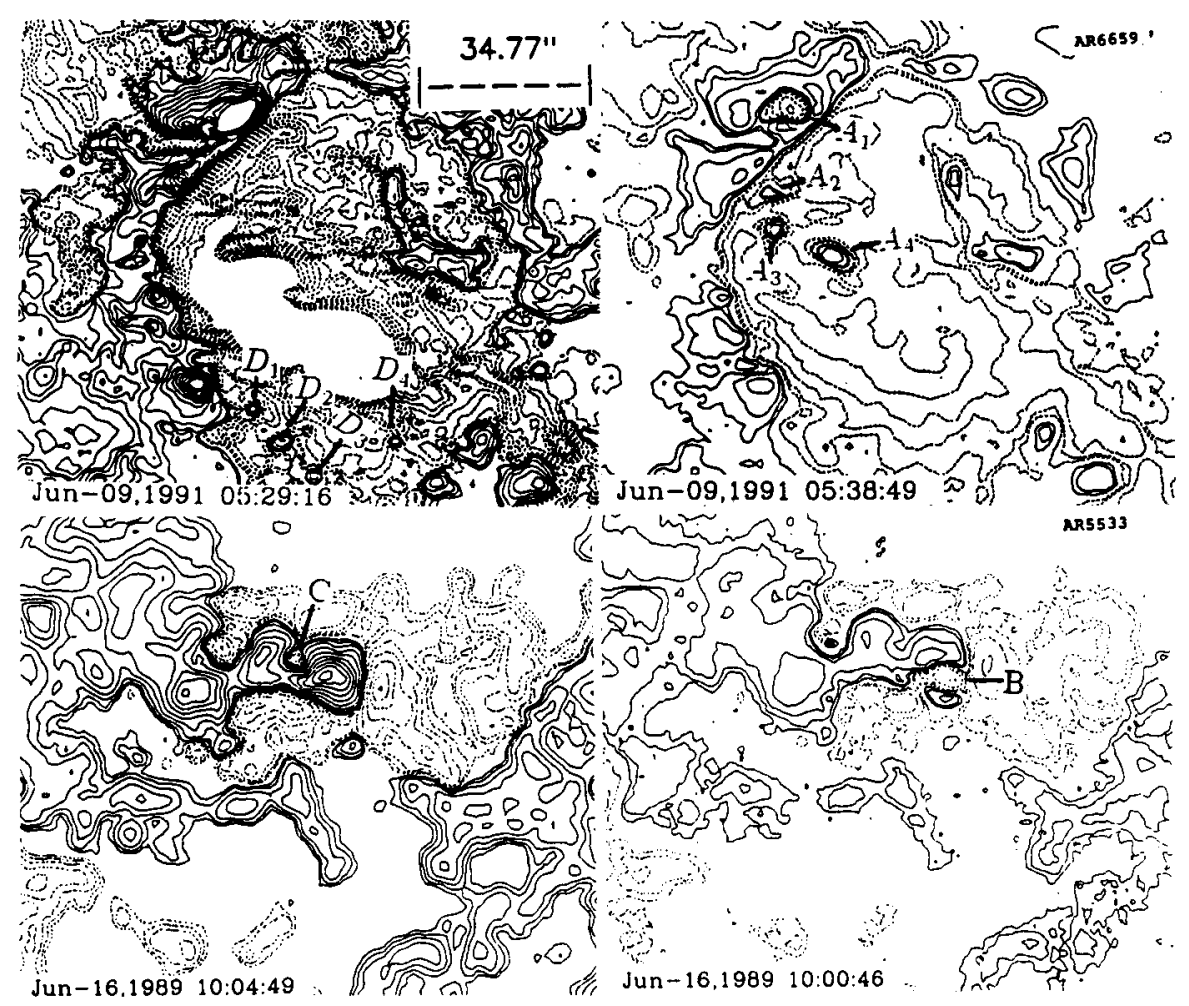

Fig. 1. Comparison of photospheric magnetograms (left) and chromospheric magnetograms (right). Solid (dashed) contours correspond to positive (negative) fields of $20,40,80,160,320,640,960,1200,1600$, 1920 Gauss. North is at the top, west is at the right.

Type B: Chromospheric magnetic gulfs of opposite polarity corresponding to photospheric fields located in the weaker magnetic fields typically found at the edges of umbra or outer edges of penumbras. Here there is the appearance of strong squeezing of the gulf by surrounding opposite polarity features in the chromosphere but not in the photosphere. The mark B in Figure 1 shows this kind of reversed structure of chromospheric magnetic field.

Type $\mathrm{C}$ is the reverse of type $\mathrm{B}$. That is, there is very strong opposite polarity magnetic squeezing in the photospheric magnetic field, but no obvious squeeze in the chromosphere. The mark $\mathrm{C}$ of Figure 1 belongs to this type.

Type $\mathrm{D}$ is the reverse of type A. Here magnetic islands were observed only in the photospheric magnetic fields. For example, the marks $D_{1}, D_{2}, D_{3}, D_{4}$ and $D_{5}$ in Figure 1 show the $\mathrm{D}$ type of reversed structures of the chromospheric magnetic fields. 
We observed 680 reversed polarity structures of chromospheric magnetic fields in 37 active regions. Of these $21.6 \%$ are type $A, 12.8 \%$ are type $\mathrm{B}, 18.8 \%$ are type $\mathrm{C}$, and $46.8 \%$ are type $\mathrm{D}$.

\section{DISCUSSION}

Opposite polarity between the chromospheric and photospheric magnetic fields has been found by many astronomers, e.g. Tsap (1971), Chen et al. (1989), Zhang (1991), Whang \& Shi (1992). We are sure of the existence of the reversed polarity structures of these chromospheric magnetic field. They are not the result of a reversed spectrum line caused by violent flare emission, and are not false appearances caused by projection or Doppler velocity effects. The reversed polarity above the photosphere represents magnetic lines of force that have been curved, twisted or knotted, or broken away from photospheric lines and then form an isolated magnetic loop in the chromosphere.

\section{ACKNOWLEDGMENTS}

We are grateful to the staff at Huairou Solar Observing Station for their support of these observations. This research was supported by the Chinese Academy of Sciences and National Science Foundation of China.

\section{REFERENCES}

Chen, J., Ai, G., Zhang, H., \& Jiang, S. 1989, Suppl. Publ. Yunnan Obs., 108 Tsap, T. 1971, in Solar Magnetic Fields, ed. R. Howard, IAU Symp. 43, 223 Whang, J. \& Shi, Z. 1992, Acta Astronomica Sinica, 33, 95

Zhang, H. 1991, presented at The First China-Japan Seminar on Solar Physics, Kunming, China. 